\title{
Clinical applications of a cognitive phonology
}

\author{
Martin J. Ball \\ From the Department of Communicative Disorders, University of Louisiana at Lafayette, USA \\ Received 30 September 2002. Accepted 8 May 2003.
}

Logoped Phoniatr Vocol 2003; 28: 63-69

It is noted that much previous work in phonology has attempted to provide economical theories of sound systems without explicitly attempting to provide theories that have psycholinguistic validity. The work of Bybee (8) on a cognitive approach to phonology is described, and its possible application to disordered speech is considered. It is discussed that cognitive phonology, coupled with gestural phonology, provides descriptive as well as explanatory accounts of disordered speech, and has specific implications for approaches to therapy. The article concludes with a case study of child with severely unintelligible speech, where it seems that the insights of cognitive phonology provide both an explanation for and a description of her speech behaviors.

Key words: child phonology, cognitive phonology, disordered speech, intervention.

Dr Martin J. Ball, Department of Communicative Disorders, University of Louisiana at Lafayette, P O Box 43170, Lafayette, LA 70504-3170, USA. E-mail: mjball@louisiana.edu

\section{APPROACHES TO PHONOLOGICAL THEORY}

As Chomsky (9) proposed, a linguistic theory has observational adequacy if it accounts for linguistic behaviors in as elegant and non-redundant way as possible, and descriptive adequacy if it accounts for the linguistic intuitions of speakers in addition to generating all and only the sentences of a language. ${ }^{1} \mathrm{~A}$ theory has explanatory adequacy if it can account for why one particular descriptively adequate grammar can be preferred over another. If we extend the meaning of this last term, we can think of a fully adequate model as an attempt to explain why particular linguistic behaviors are chosen. In other words we can look for psycholinguistic validity in a theory; an attempt to model how the brain organizes particular linguistic behaviors and operationalizes them. Such a division in types of adequacy applies to models of phonology just as much as to other areas of linguistic organization.

Early generative models of phonology, for example, Chomsky and Halle (10), strove to achieve descriptive adequacy, but did not claim the sort of psycholinguistic adequacy we described above. Developments such as autosegmental and metrical phonology described

\footnotetext{
${ }^{1}$ An earlier version of this paper was presented at the 9th Symposium of the international Clinical Phonetics and Linguistics Association, Hong Kong, May 2002.
}

by Goldsmith (15), and feature geometry described by Clements (11), can all be seen as attempts to produce more elegant descriptions of phonological phenomena. Nevertheless, even within the generative school, approaches such as underspecification, see Archangeli (1), were often justified by appealing to psycholinguistic plausibility. It was argued, for example, that underspecified segments would take less storage space than fully specified ones. Note, that such an argument assumes the necessity for economic neurolinguistic storage, whereas evidence from recovery from aphasia (among other phenomena) suggests that, in fact, multiple redundant storage may better characterize language in the brain (see Code's (12) review of this area).

The generative enterprise was not the only force in phonology in the last 30 years, however. Stampe and colleagues (13) argued in favor of a 'Natural Phonology' that was explicitly innatist: in other words a search for explanatory adequacy. Since Stampe, we have seen a variety of models of phonology based to a lesser or greater extent on theory external validity. For example, Articulatory (or Gestural) Phonology $(4,5)$ is based on articulatory gestures (and so ultimately motor programs); Government Phonology $(17,18)$ on acoustic patterns; and Sonority theory (23) on perception.

Generative Phonology and developments from it have, nevertheless, claimed as psycholinguistically 
valid the notion of derivation. The many surface realizations of pronunciations are derived from a few underlying stored forms. Allophonic and morphophonological variants are derived by rule from underlying forms. This results in fewer forms needing to be stored in the brain, along with a set of rules to convert the abstract underlying forms into actual realizations. While this seems plausible psycholinguistically, even the notion of derivation has been challenged by some theorists, such as those working with Optimality theory (2), and with cognitive phonology (8). Clearly, when working with clinical data, a phonological approach that is psycholinguistically plausible may be of more use in informing intervention, than one that has descriptive elegance but not explanatory force.

\section{COGNITIVE PHONOLOGY}

Coming out of functional and cognitive linguistics, Joan Bybee (8) has recently outlined a model of what we will term here Cognitive Phonology. ${ }^{2}$ As the name suggests, this approach is explicitly designed to model phonology as a cognitive activity, and so to demonstrate the kind of psycholinguistic approach we have been discussing. It is also a radical departure from previous approaches, as we will outline below. ${ }^{3}$

\section{Hypotheses of Cognitive Phonology}

Among the important hypotheses of Cognitive Phonology, as described in Bybee (8), are those involving frequency of use. Bybee notes that experience affects representation, in that high frequency forms and phrases have stronger representations in memory, and so they are resistant to analogic change. Low frequency forms are more difficult to access, and may be subject to change or loss. Patterns (schemas: to be described below) that apply to more items are also stronger and more productive.

Cognitive Phonology also claims that mental representations of linguistic objects have the same properties as mental representations of other objects. The result of this claim is that mental representations of linguistic objects do not have predictable properties abstracted away, but are based on categorization of actual tokens. Such a claim is completely opposed to

\footnotetext{
${ }^{2}$ Note that Bybee (8) does not actually call her approach 'cognitive phonology', preferring to avoid at this stage specifying her account too strictly.

${ }^{3}$ There are several other functional approaches to phonology, e.g. Systemic-Functional, see Tench (21), and the Columbia school, see Tobin (22). These also have possible clinical applications, but we restrict ourselves to Bybee's approach in this article.
}

the accepted wisdom of generative models, where predictable properties of units are posited and are abstracted away via the process of derivation. As we noted above, underspecification approaches are claimed to have psycholinguistic validity through the minimization of storage space: Cognitive Phonology opposes this view. Derivation and underspecification are viewed as simply attempts to achieve descriptive elegance; Cognitive Phonology does not object to redundant storage: indeed, it claims that this is in fact what happens. Generalizations over forms are not separate from the stored representations of forms, according to the cognitive approach, but emerge directly from them. Generalizations are expressed as relations among forms based on phonetic/semantic similarities. So, multimorphemic words are stored whole in the lexicon (therefore non-derivationally).

Cognitive Phonology also states that categorization is based on identity or similarity. Categorization organizes the storage of phonological percepts, as we will exemplify below. The theory does not specify any one approach to phonological units, but Bybee (8) tends toward the Gestural approach of Browman and Goldstein $(4,5)$, as exemplified for clinical data by Kent (19). ${ }^{4}$ It is worth noting that Browman and Goldstein (6) actually link their approach to phonology into a collection of work on language and cognition. Here, they tie gestural phonology into a dynamical approach to cognition, according to which cognitive processes are the behavior of nonlinear dynamical systems, and can best be studied using dynamical modeling and dynamical systems theory. Other units such as morpheme, syllable, phoneme/ segment are not basic units of the theory, but are emergent: they arise from the relations of identity and similarity that organize stored units. In other words, as links between similar items in storage become stronger (due to multiple examples and to frequency of access to items), these items - which may be phoneme-sized, morpheme-sized, etc. - become stronger, and emerge as units of storage. Storage in Cognitive Phonology is highly redundant (as opposed to the attempts at descriptive elegance of other approaches), so schemas may describe the same pattern at different degrees of generality.

Finally, we can note that the theory claims that grammatical knowledge is procedural knowledge. Phonology becomes a part of the procedure for producing and decoding constructions, rather than a purely abstract, psychological system. This model, then, is not looking for descriptive economy, but for psycholinguistic plausibility.

\footnotetext{
${ }^{4}$ We illustrate the use of Gestural Phonology below, in the description of the case study.
} 


\section{Organized storage}

Generative models claim that material appearing in rules cannot appear in the lexicon and vice versa: this underlies the whole principle of derivation. Cognitive Phonology explicitly rejects this, and claims that predictable features do appear in schemas (patterns, rather than rules), but also in the lexicon. This means that words and phrases can be stored whole, yet still participate in the schemas that link similar forms. This is because storage is not linear but spatially networked (see Figs. 1-3).

The three figures give examples of schemas linking phonological and morphological forms in the lexicon through networks. As noted, the strength of these networks is important and derives in part from frequency of use. Units such as phonemes and morphemes emerge from these schemas. The strength of connections also depends upon the degree of similarity, and this point is explored more fully in Bybee (7).

Schemas are non-process statements about stored items, and can be thought of as generalizations about linguistic units that emerge from the activity of categorizing linguistics items for storage by speakers. Bybee (8) describes four characteristics of schemas. First, schemas are patterns of organization in the lexicon, and so have no independent existence apart from the lexical units from which they emerge. Secondly, schemas can be more or less productive dependent upon the number of items linked to the schema. Thirdly, the phonological shape of the prototype exemplar of a particular class affects the members of that schema, although, finally, schemas can be gradient categories, with individual types closer or further from the best exemplars.

To better illustrate schemas, we can consider three such generalizations emerging out of linguistic storage in English.

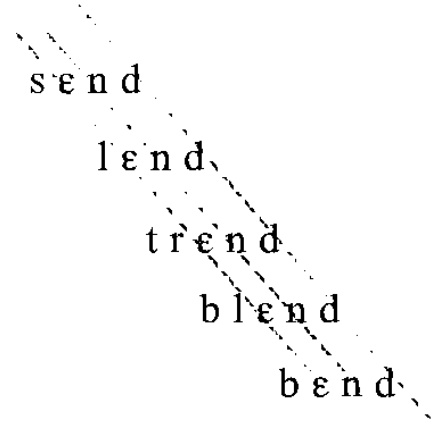

Fig. 1. Lexical connections for [end] in send, lend, trend, blend, bend.

$$
\begin{aligned}
& \text { p l e i d dpàst] } \\
& \text { s p I l d [past] } \\
& \text { s p o I } 1 \text { d [pàst] } \\
& \begin{array}{l}
\mathrm{b} æ \mathrm{n} \mathrm{d} \quad \text { [pàșt] } \\
\mathrm{r} æ \mathrm{~m} \text { d }
\end{array}
\end{aligned}
$$

Fig. 2. Phonological and semantic connections yield Past in played, spilled, spoiled, banned rammed.

$$
\begin{aligned}
& \text { spo } 1 \\
& \text { spo I l I } \\
& \text { p l e I } \\
& \text { p l e i i g } \\
& \mathrm{r} \mathfrak{x} \mathbf{m} \\
& \text { r } \text { m I } \\
& b æ n \\
& \mathrm{~b} \mathfrak{x} \mathrm{n} \text { I } \mathrm{y}
\end{aligned}
$$

Fig. 3. The emergence of the -ing suffix in spoil, spoiling; play, playing; ram, ramming; ban, banning .

1. [ $[\bar{\varepsilon} \mathrm{nd}]$ is a possible syllable rhyme: [\$_ēnd \$] (This is diagrammed in Fig. 1).

2. [[VERB] d] means Past Tense: [[VERB] d]Past Tense (This is diagrammed in Fig. 2)

3. [[VERB]In] means Present Progressive: [[VERB] Iy] Present Progressive (This is diagrammed

So, what in generative models are derivational rules, in Cognitive Phonology are schemas formed by links between forms in the lexicon. Cognitive Phonology is, therefore, very 'flat' in terms of derivation, but multidimensional in terms of associations, as virtually all realizations are stored lexically. This differs, then, from most other approaches to phonological description, where the rule or process is an important device to link different levels of abstraction or description. Nevertheless, Cognitive Phonology, as described by Bybee (8) does recognize processes. Two main types of processes are recognized: fast speech processes, and historical change.

Fast speech processes are deemed to be lenitions (weakenings) accounted for by reduction and retiming of articulatory gestures as described in gestural models of phonology. Historical changes come about through the mechanisms of lenition and analogy. An example of historical change through lenition is the change 
from $[\mathrm{s}]$ to $[\mathrm{h}]$ word finally in certain South American varieties of Spanish. Analogic change can be exemplified by spelling pronunciations (such as the pronunciation of $/ \mathrm{h} /$ at the beginning of herb in British English) or the regularization of past tenses in forms such as wept - weeped, and spelt-spelled. The effect of analogy can be blocked by high-frequency use, whereas highfrequency use encourages lenition.

\section{APPLICATION OF COGNITIVE PHONOLOGY TO DISORDERED SPEECH}

Can such a model be applied to the various patterns of disordered speech encountered in the clinic and, if so, what implications does this have for assessment, diagnosis and intervention? We can, therefore, imagine three possible approaches related to these themes: descriptive, explanatory, and remediation planning.

\section{Descriptive}

If units such as phonemes and syllables are emergent, and processes are either lenition or analogy, then we would need to describe the disordered data differently in a Cognitive Phonology approach than in other popular clinical assessment types such as Natural Phonology (16). Fundamentally, therefore, we would need to describe data in terms of weak or non-existent lexical networks first, leading to specific problems with segments or other units. When patterns of lenition are observed (such as in dysarthric speech) the formalisms of gestural phonology can be employed in descriptions of the clinical data. However, it should be remembered that some child phonological disorders (and patterns of normal phonological development) appear to be more readily described in terms of fortitions (such as fricative stopping). These, of course, can be accounted for through the same gestural formalisms. We should also note that Bybee's model does allow for different processes in child phonological development.

\section{Explanatory}

If there is inadequate lexical storage, leading to networks that are not strongly enough established and linked, then units (phonemes/morphemes, etc) will not emerge. So, we can assume that problems arise if insufficient items are stored correctly in the lexicon, or if the categorization that creates connections does not occur. (For example, an interpretation within Bybee's (7) model of morphology of what happens in SLI that prevents the development of morphology is that morphologically complex words are not categorized to form the associations required.) Of course, it may be difficult to know whether lexical storage is faulty because there simply are not enough items stored to create the proper networks and allow units to emerge, or because some or many items that are stored, are stored incorrectly, thus disrupting expected networks.

The patterns of substitutions and deletions we see in disordered speech need also to be explained. As we have just noted, Cognitive Phonology lays stress on lenition as the main (or only) process of synchronic change. However, we have already commented that some disordered speech does not show lenition, but rather fortition. This can be accounted for within Bybee's account by appealing to analogy: unestablished networks (unestablished due to insufficient or inaccurate storage) are replaced by those that $d o$ exist. The existing networks may have come about due to correct storage of a dominant pattern (e.g., when stops are realized instead of fricatives because the stop networks are stronger), or incorrect storage (e.g., when sounds from outside the target system are used, as in [1] for English /s/). What caused that incorrect storage is, of course, a further step back in the diagnosis process. Bybee (8) discusses possible causes of sound changes, noting that a plausible explanation of changes that affect low frequency forms first (such as front rounded vowels in earlier forms of English) could well be the result of incorrect perception of the form during acquisition. As these forms are rarely heard, there are few examples for the child to correct the misperception. The other main source of sound change is re-arrangement of articulatory gestures (usually lenition). This affects high frequency forms most, as there is the greatest benefit to the user to simplify forms that are used most often. In disordered speech, therefore, we may see incorrect storage due to perceptual breakdown or due to articulatory difficulty (or a combination of the two).

\section{Planning remediation}

In Bybee's account of Cognitive Phonology (8), frequency of use is much more important than notions of contrast. Therefore, in remediation, minimal pairs drills, the staple of many other approaches to remediation that do stress contrast, would not, it is assumed, be deemed of major importance. More important would be drilling sets of words that would reinforce networks and allow specific units to emerge.

\section{EXAMPLE}

In Ball, Müller and Damico (3) the authors report on a 6-year-old girl who presented with severely unintelligible speech. 'Susan' had been receiving speech therapy since age 2;6 at home and in the school system. She had a significant ENT history, coupled 
with asthma and Attention Deficit Disorder (ADD). Tonsillectomy, adenoidectomy and tympanostomy were performed at 3;9 and pressure equalization (PE) tubes (also known as ventilation tubes or grommets) were inserted bilaterally. The oral muscular examination was unremarkable except for a Class III Malocclusion (underbite). Temporomandibular joint dysfunction was identified at $4 ; 9$, though this had no adverse effect on mastication, swallowing or speech. Susan was prescribed daily doses of the antihistamine Loratadine for asthma triggered by allergic responses, and a stimulant medication for ADD. She had had an unsettled home background, and was currently living with her grandparents. On entry to the University of Louisiana at Lafayette clinic, baseline scores were obtained on the Goldman-Fristoe Test of Articulation (14) of 37 errors on the sounds in words subtest and 29 errors on the sounds in sentences subtests. The KhanLewis phonological analysis (20) gave a composite score of 20 , and an age equivalency of $3 ; 9$.

Initial assessment of Susan's speech showed she was stimulable for virtually all the contrastive segments of English. However, this did not carry over into spontaneous connected speech which was characterized by the use of glottal stops in syllable onsets and codas, intact vowels and intact prosody. In her connected speech, final consonants occurred correctly more often than initial, and bilabial more often than other places of articulation; glottal stop realizations were, however, the most common.

Particularly interesting behavior occurred during a syllable repetition task undertaken to test Susan's stimulability $^{5}$ for a selection of consonant-vowel combinations. The task involved the client repeating a series of nonsense syllables that were modeled by the clinician. Susan adopted four different strategies in this task: three of which involved the use of a process for which the authors introduced the term double onsets (for a justification of this term see Ball et al, 3). These strategies, with typical examples of their realizations, were:

1. Repeat onset: [h: (.) hu] (target $/ \mathrm{hu} /)^{6}$

2. Denasalization: [m: (.) bu] (target $/ \mathrm{mu} /)$

3. Glottal replacement: [s: (.) $7 \mathrm{u}]$ (target /su/)

4. Correct: $[\mathrm{tu}]($ target $/ \mathrm{tu} /)$

The exercise involved the following consonants in this order: /h, m, n, s, $\int, 1, \mathrm{r}, \mathrm{t} /$ which were followed by all

\footnotetext{
${ }^{5}$ In this usage, stimulability means the ability of the client to imitate modeled forms.

${ }^{6}$ The length mark in the transcriptions show the drawn out nature of the first consonant; the period in parentheses denotes the very short pause between the onsets.
}

the monophthongal vowels of English. The test forms were modeled in consonant order (i.e., all the $/ \mathrm{h} /+$ vowel forms were modeled before the $/ \mathrm{m} /+$ vowel forms were started, and so on). Susan used correct forms for all these consonants, although normally these only occurred after one or more corrections by the clinician. The exception was $/ t /$, where correct forms predominated, with repeat onset as the other main variant. With $/ \mathrm{h} /$, repeat onset was the dominant form. The two nasals showed denasalization most commonly, but repeat onset was also quite common, followed by the occasional use of the glottal realization. The two fricatives most commonly demonstrated glottal replacement, though some instances of repeat onset also occurred. The liquids both presented problems to Susan in that she was not able to produce consistently acceptable realizations. Her onset strategies appeared to be repeat onset and glottal realization, but the repeated forms were not always similar or indeed close to target (the first onset was often schwalike, and the second glide-like).

Applying the approach to phonology outlined earlier in this article, it is possible to describe Susan's realizations in both the repetition task and spontaneous speech through a rearrangement of gestures (using the formalisms of gestural phonology). Figs. 47 show the gestural scores (as they are termed in this approach) for the target forms and for the realizations

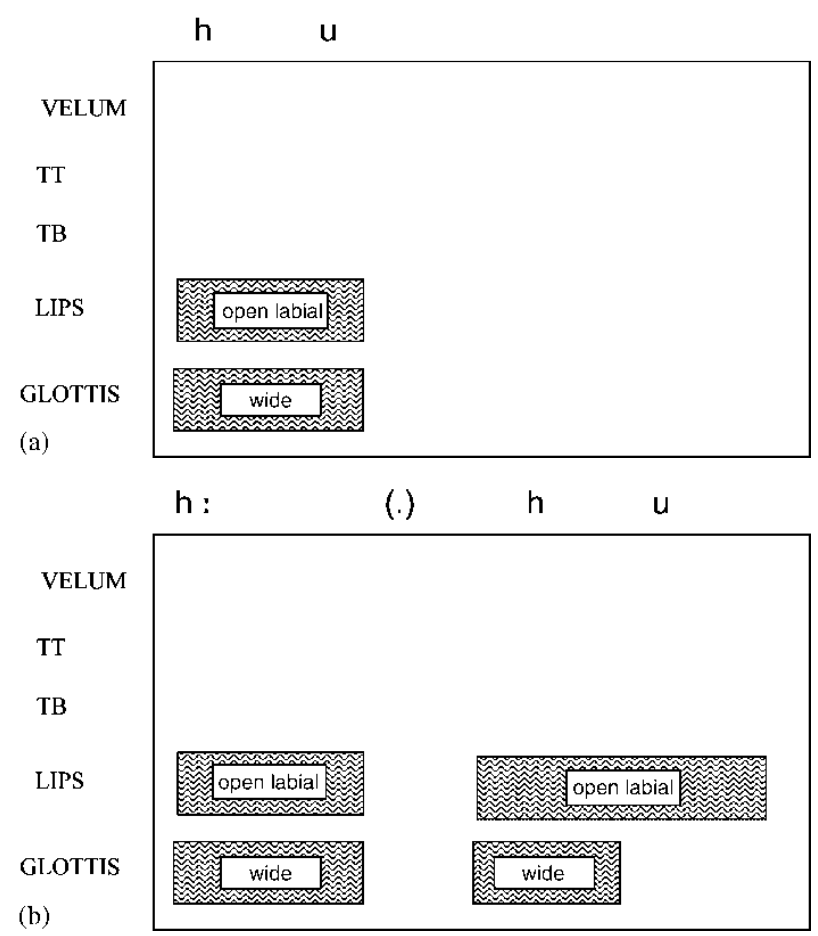

Fig. 4. a). Gestural Score for target [hu]; b) Gestural Score for [h: (.) hu]; TT - tongue tip, TB - tongue blade. 


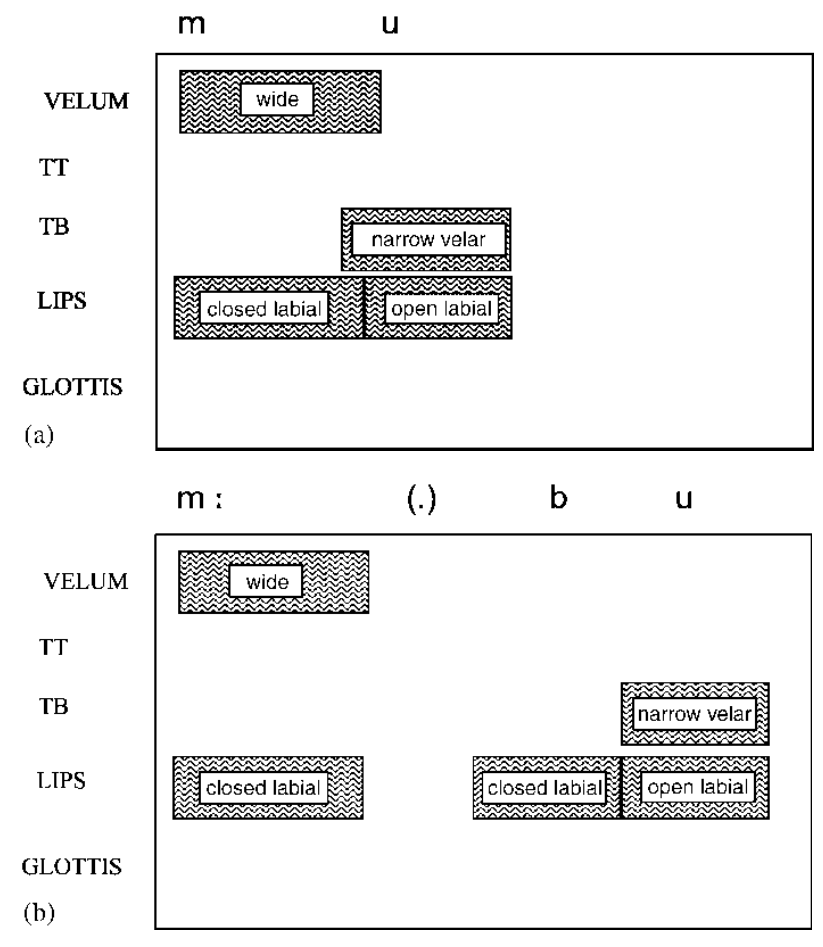

Fig. 5. a). Gestural Score for target [mu]; b) Gestural Score for [m: (.) bu]; TT - tongue tip, TB - tongue blade.

S

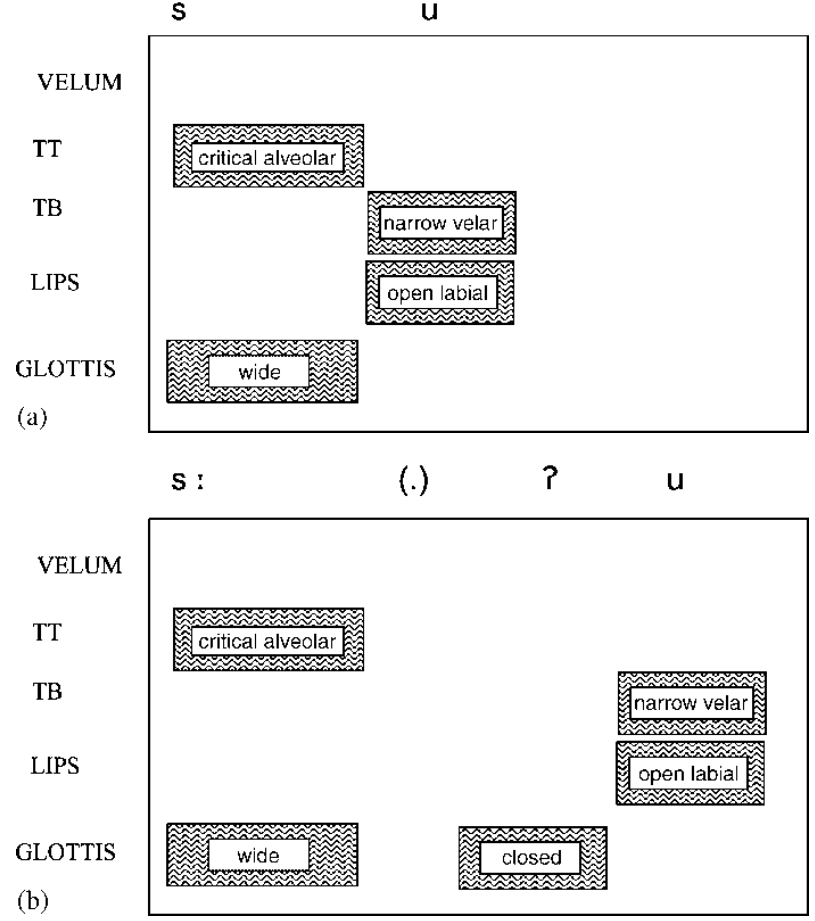

Fig. 6. a). Gestural Score for target [su]; b) Gestural Score for [s: (.) ?u]; TT - tongue tip, TB - tongue blade.

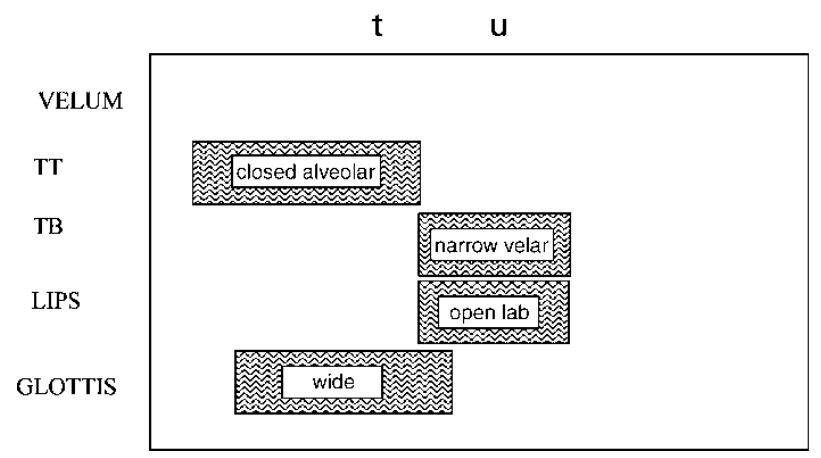

Fig. 7. Gestural Score for target and realization [tu]; TT - tongue tip, TB - tongue blade.

of the four strategies adopted by Susan. Fig. 4 shows repeated [h], and the score demonstrates the complete simplicity (in gestural terms) of the target $/ \mathrm{h} /$, which needs just a wide glottal gesture. Fig. 5 demonstrates the strategy of denasalization, and we see that in this instance the score is simplified by the removal of the velum gesture. Fig. 6 shows the process of glottal replacement, and we see here a further simplification in that the tongue tip gesture is removed, and the glottis gesture is reconfigured from wide to closed. In this respect, the score for [h] and for [?] are, of course, similar, apart from the width of the glottal gesture.. Finally, Fig. 7 shows the score for a correct, unrepeated $/ \mathrm{t} /$. This demonstrates that the problematic gesture for /s/ was not the tongue tip per se, but the constriction of that gesture: closed (as for / $t /$ ) presents no problem, but 'critical' (i.e., in a position to make a grooved fricative) is not available in spontaneous speech. Therefore, Susan replaces this with a closed glottis gesture. This latter is the most simple of gestures, as all the other gestures (velum, tongue tip, tongue body, and lips) can be ignored. This strategy is also commonly used in her spontaneous speech as noted above.

The behaviors described here can arguably be thought of as examples of lenition, at least within gestural phonology terms. This is because denasalization and glottal replacement all involve the loss of certain gestures or weakening of the overall gestural score. Traditional schools of phonology have not recognized reduction to glottal stop or denasalization as lenition processes, however. Nevertheless, as noted earlier, the precise nature of processes in disordered speech do not have to adhere to the proposals made for normal phonology. In terms of the explanatory section above, Susan's problems could well be a result (at least partly) of her medical problems in that disruptions to hearing and attention could have resulted in incorrect storage due to perceptual disruptions. Her malocclusion and temporomandibular pro- 
blems may also have resulted (at least at an early stage) in difficulties with articulation which themselves (as was noted above) may result in incorrect storage. The notion of density of storage suggested by Bybee's work is also seen in the fact that Susan can produce target phonological realizations when prompted, but rarely in spontaneous speech. This implies that these forms are stored, but that substitution forms have stronger links and so are accessed more readily.

As discussed earlier, a cognitive phonology approach suggests that contrast is less important in phonology than the reinforcement of networks containing sounds and sequences of sounds that were problematic for the client. In the case of Susan the syllable repetition task outlined earlier can be thought of not only as a data collection strategy, but also as a drill to emphasize sets of consonant-vowel networks needed for the target phonology. Unfortunately, it proved impossible to track the client's progress as, after some initial improvement occurred, she was withdrawn from therapy at the University clinic due to travel problems and resumed therapy through the school system. Nevertheless, Bybee's model (8) of phonological organization coupled with the insights of gestural phonology, seem to account for Susan's phonological realizations and to suggest an underlying cause for them.

\section{CONCLUSION}

Cognitive Phonology is a refreshingly different approach to the psycholinguistic organization of speech. Many of its insights seem valuable; and some of these insights appear applicable to phonological acquisition and disorders in a direct way. Clearly, research comparing intervention techniques based on cognitive phonology and those based on traditional approaches, with large numbers of clients is needed to test these ideas.

\section{ACKNOWLEDGEMENTS}

I would like to thank Joan Bybee for most helpful comments on an earlier draft of this paper. All remaining shortcomings are, of course, my responsibility.

\section{REFERENCES}

1. Archangeli D. Aspects of underspecification theory. Phonology 1988; 5: 183-207.
2. Archangeli D, Langendoen T. Optimality Theory. An Overview. Oxford: Blackwell; 1997.

3. Ball MJ, Müller N, Damico H. Double Onset Syllable Structure in a Speech Disordered Child. Advances in Speech-Language Pathology 2003; 5: 37-40.

4. Browman C, Goldstein L. Towards an articulatory phonology. Phonology Yearbook 1986; 3: 219-52.

5. Browman C, Goldstein L. Articulatory phonology: an overview. Phonetica 1992; 49: 155-80.

6. Browman C, Goldstein L. Dynamics and articulatory phonology. In: Port R, van Gelder T (eds). Mind as Motion. Explorations in the dynamics of cognition. Cambridge, Mass.: MIT Press; 1995. pp. 175-93.

7. Bybee J. Morphology: A Study of the Relation Between Meaning and Form. Amsterdam: John Benjamins; 1985.

8. Bybee J. Phonology and Language Use. Cambridge: Cambridge University Press; 2001.

9. Chomsky N. Aspects of the Theory of Syntax. Cambridge, MA: MIT Press; 1965.

10. Chomsky N, Halle M. The Sound Pattern of English. Cambridge, Mass.: MIT Press; 1968.

11. Clements G. The geometry of phonological features. Phonology Yearbook 1985; 2: 225-52.

12. Code CF. Language Aphasia and the Right Hemisphere. Chichester: John Wiley; 1987.

13. Donegan P, Stampe D. The study of Natural Phonology. In: Dinnsen D (ed.). Current Approaches to Phonological Theory. Bloomington: Indiana University Press; 1979. pp. 126-73.

14. Goldman R, Fristoe M. Goldman-Fristoe Test of Articulation. Circle Pines, MN: American Guidance Service, Inc; 1986.

15. Goldsmith J. Autosegmental and Metrical Phonology. Oxford: Blackwell; 1990.

16. Grunwell P. Natural phonology. In: Ball MJ, Kent RD (eds). The New Phonologies. San Diego: Singular; 1997. pp. $35-75$.

17. Harris J, Lindsey G. The elements of phonological representation. In: Durand J, Katamba F (eds). Frontiers of Phonology. London: Longmans; 1995. pp. 3479.

18. Harris J, Lindsey G. Vowel patterns in sound and mind. In: Burton-Roberts N, Carr P, Docherty G (eds). Phonological Knowledge, Conceptual and Empirical Issues. Oxford: Oxford University Press; 2000. pp. 185-205.

19. Kent RD. Gestural phonology: basic concepts and applications in speech-language pathology. In: Ball M J, Kent RD (eds). The New Phonologies. San Diego: Singular; 1997. pp. 247-68.

20. Khan L, Lewis N. Khan-Lewis Test of Phonological Analysis. Circle Pines, MN: American Guidance Service, Inc; 1986.

21. Tench P, editor. Studies in Systemic Phonology. London: Pinter Publishers, 1992.

22. Tobin Y. Phonology as Human Behavior. Durham: Duke University Press; 1997.

23. Yavas M. The role of sonority in developing phonologies. Journal of Multilingual Communication Disorders 2003; 1: 79-98. 
Copyright $\odot 2003$ EBSCO Publishing 